\title{
THE COLORS OF COLLOIDS. II
}

BY WILDER D. BANCROFT

\section{Reflection and Refraction}

When a beam of light strikes a plane, polished surface, a portion of the light is reflected in such a way that the angle of incidence, measured from the normal to the surface, is equal to the angle of refraction. The remaining portion of the beam is refracted and passes through the surface in such a way that the ratio of the sine of the angle of the incident ray is proportional to the sine of the angle of the refracted ray, or sin $i / \sin r=$ constant. This is known as Snell's law. It was discovered in I62I and holds for isotropic media only. The constant in the equation is the relative refractive index between the media, and the equation holds for every value of $i$, if the second medium is the one in which the light travels at a lesser velocity. While a refracted ray always exists when the light passes from an optically less dense to an optically denser medium, no matter how great the angle of incidence, the converse is not always true. If the relative refractive index is less than one, $\sin r$ should be greater than one when $i$ exceeds a certain angle. No angle has a sine greater than one and therefore there can be no refracted ray and we are dealing with total reflection. The angle at which this occurs is called the critical angle. Tyndall ${ }^{1}$ says that "In all cases when the light is incident from air upon the surface of a solid or a liquid, or, more generally still, when the incidence is from a less highly refracting to a more highly refracting medium, the reflection is partial. In this case the most powerfully reflecting substances either transmit or absorb a portion of the incident light. At a perpendicular incidence water reflects only i 8 rays out of every rooo; glass reflects only 25 rays, while mercury reflects 666 . When the rays strike the surface obliquely the reflection is augmented. At an

\footnotetext{
1 "Six Lectures on Light," I7 (1883).
} 
incidence of $40^{\circ}$, for example, water reflects 22 rays, at $60^{\circ}$ it reflects 65 rays, at $80^{\circ} 333$ rays; while at an incidence of $89.5^{\circ}$ where the light almost grazes the surface, it reflects 721 rays out of every rooo. Thus as the obliquity increases the reflection from water approaches, and finally quite overtakes, the reflection from mercury; but at no incidence, however great, when the incidence is from air, is the reflection from water, mercury, or any other substance, total. Still total reflection may occur, and with a view to understanding its subsequent application in the Nicol's prism, it is necessary to state when it occurs..... Total reflection never occurs except in the attempted passage of a ray from a more refracting to a less refracting medium; but in this case, when the obliquity is sufficient, it always occurs. Total reflection may be illustrated simply by placing a shilling in a drinking glass and tilting the glass so that the light from the shilling shall fall with the necessary obliquity upon the water surface above it. Look upwards toward that surface and you see the image of the shilling shining as brightly as the shilling itself. Thrust the closed end of a glass test-tube into water, and incline the tube. When the inclination is sufficient, horizontal light falling upon the tube cannot enter the air within it, but is totally reflected upward; when looked down upon such a tube looks quite as bright as burnished silver. Pour a little water into the tube; as the liquid rises, total reflection is abolished, and with it the lustre, leaving a gradually diminishing, shining zone, which disappears wholly when the level of the water within the tube reaches that without it. Any glass tube, with its end stopped water-tight, will produce this effect, which is both beautiful and instructive."

Total reflection will not occur if the medium having the lower index of refraction is present as a thin film." "Bring a convex surface of glass of large radius of curvature into contact with the surface at which total reflection is taking place. The light will be found to enter the lens in an annular region surrounding the point of contact. This is due to the fact

1 Wood: "Physical Optics," 373 (r9r r). 
that the two glass surfaces come into optical contact, $i$. e., we may regard the air film as squeezed out completely. This circular patch over which total reflection fails and complete transmission obtains, is surrounded by a ring which transmits light of a reddish and reflects light of a bluish tinge. The glass surfaces are not in contact here; but the air film is too thin for total reflection to take place. Transmission will obviously occur for red light first, since the thickness of the film necessary to reflect light is measured in comparison with the wave-length."

Wood ${ }^{1}$ points out that while "the velocity of light in the free ether of space is independent of the color or wave-length, such is not the case in refracting media. Here the waves not only travel slower than in free space, but waves of different length travel with very different velocities. In all such media as air, water, and glass, the long waves travel faster than the short ones; consequently the deviation of the ray, or the angle through which the wave-front turns when encountering the boundary of the medium, depends on the color of the light as well as on the optical density of the medium."

"When white light enters a transparent medium, the long red waves forge ahead of the green ones, which in their turn get ahead of the blue. If we imagine an instantaneous flash of white light traversing a refracting medium, we must conceive it as drawn out into a sort of linear spectrum in the medium, that is, the red waves lead the train, the orange, yellow, green, blue, and violet following in succession. The length of this train will increase with the length of the medium traversed. On emerging again into free ether the train will move on without further alteration in its length.

"We can form some idea of the actual magnitude involved in the following way: Suppose we have a block of perfectly transparent glass (of refractive index 1.52) twelve miles in thickness. Red light will traverse it in $I / 10000$ of a second. If white light were to traverse this mass of glass, the time elapsing between the arrival of the first red and the first blue

I "Physical Optics," 9I, Ior (IgII). 
light would be less than $\mathrm{r} / 6000$ of a second. Michelson's determination of the velocity of light in carbon bisulphide showed that the red waves gained on the blue waves during their transit through the tube of liquid. The absence of any change of color in the variable star Algol furnishes direct evidence that the blue and red rays traverse space with the same velocity. In this case the distance is so vast, and the time of transit so long, that the white light coming from the star during one of its periodic increases in brilliancy, would arrive at the earth with its red components so far in advance of the blue that the fact could easily be established by the spectrophotometer or even by the eye.

"Inasmuch as the deviation of a ray of light depends on the change of velocity of a wave on going, say, from a rare into a denser medium, we infer that those rays which are deviated the most, namely the violet, suffer the greatest change of velocity or move the slowest...... In addition to the more or less regular gradation in the refractive index of the atmosphere there exist striae, or small regions of sudden change due to the mixing of hot and cold currents, somewhat similar to the conditions existing in a mixture of glycerine and water. When a wave-front of light passes through a region where the refractive index is low, it gains; and it loses while travelling in a region of high refractive index. The result of this is that the striae deform the plane-waves of light coming from the stars into corrugated waves, portions of which are convex in the direction of propagation, while other portions are concave. The concave portions naturally converge, while the convex portions diverge, the result being that the energy concentrates itself in certain areas at the expense of the adjacent areas. This uneven distribution of light produces the familiar phenomenon of scintillation or twinkling of the stars. If the intensity of the light from a star were sufficient, we should find that instead of illuminating a white surface uniformly, as does the sun or the moon, it would illuminate it unevenly, dark and light patches alternating over the surface. This uneven illumination is actually ob- 
served during the few moments immediately preceding the total stage of a solar eclipse, the patches of light being arranged in more or less parallel bands."

When a plane wave passes through a glass plate bounded by parallel planes, ${ }^{1}$ the emergent wave-front is parallel to the incident wave-front, no matter how great the angle of incidence. The direction of the ray is therefore shifted to one side by its passage through the plate. In the case of a prism, the emerging ray is deviated towards the base of the prism and is therefore not parallel to the incident ray. The amount of deviation depends on the index of refraction and the angle of the prism. Since the index of refraction is ordinarily greater the shorter the wave-length, the violet rays will be deviated the most and the red rays the least. The degree of dispersion or the length of the spectrum depends upon the differences in the refractive indices for the different rays and is therefore not necessarily greater the higher the refractive index of the prism material, though this is often the case.

"In the case of transparent substances the dispersion is said to be normal, that is, the refractive index increases as the wave-length decreases, though the rate of change varies according to the nature of the substance. In the case of substances which show selective absorption this is generally not the case, the refractive index for the short waves in the blue side of the absorption band being less than the index for the red light on the other side of the band. This phenomenon has been named anomalous dispersion; but there is nothing anomalous about it, the so-called normal dispersion being nothing more than a special case of the anomalous. Fox Talbot appears to have been the first to notice the peculiar effect, but his discovery was not followed up. In I 860 Le Roux ${ }^{2}$ discovered that a prism containing iodine vapor deviated the red rays more than the blue, the indices at a temperature of $700^{\circ} \mathrm{C}$ for the red and violet being 1.025

1 Wood: "Physical Optics," 68 (Igr).

${ }^{2}$ Ann. Chim. Phys., [3] 4I, 285 (186I). 
and 1.019. Christiansen ${ }^{1}$ detected anomalous dispersion in the case of an alcoholic solution of fuchsine, which is one of the aniline dyes having a strong absorption band in the green. Of the remaining colors, the red, orange, and yellow occur in the same order as in the case of a glass prism. The violet, however, is less refracted than the red and separated from it by a dark interval. Christiansen's prism was made of two glass plates inclined at an angle of one degree, the solution being held between them by capillarity. The subject was next investigated by Kundt. ${ }^{2}$ His observations showed that the phenomenon is to be observed in the case of all bodies which reflect certain wave-lengths selectively. Ordinary pigments do not belong to this class, the color being produced" by absorption."

Since the transmission of light through a material medium. is always accompanied by absorption, we shall get different degrees of absorption with varying thickness of the absorbingmedium." "If the absorbing medium is homogeneous, the quantity of light of a given wave-length which is absorbed. will be proportional to the thickness of the medium traversed. If we represent the intensity of the light that enters the front surface of the medium by $I$, the intensity after transmission. through unit thickness can be represented by $I a^{e}$, in which $a$ is a fraction depending on the nature of the medium and the wave-length of the light. If the same fraction is absorbed by each successive layer, it is clear that the intensity, after traversing a thickness $\epsilon$ of the medium, will be $I a^{\epsilon}$, the quantity of $a$ being called the coefficient of transmission. The coefficient of transmission varies with the color, and the emergent light is therefore colored. In the case of most absorbingmedia the color of the transmitted light does not depend to any great degree on the thickness, the depth or saturation: merely increasing. In some cases, however, the color depends: on the thickness, thin layers, for example, appearing green,

\footnotetext{
${ }^{1}$ Pogg. Ann., 4I, 479 (I870); I46, I54 (I872).

2 Ibid., I42, I63; I43, I49, 259; I44, I28 (I87I); I45, 67, I64 (I872).

3 Wood: "Physical Optics," 438 (IgII).
} 
and thick layers red. Such substances are said to exhibit dichromatism. Some of the aniline dyes, or mixtures of them, show the phenomenon. Thin layers of a solution of cyanine appear blue, thick layers red. The addition of a little nitrosodimethyl aniline to the solution gives us a greenred dichromatic liquid, as has been shown by Pflüger. The explanation of the change of tint is very simple. Suppose we have a substance which absorbs the yellow and blue. The transmitted light then consists of a mixture of red and green. Let us assume, as is usually the case, that the intensity of the green is greater than that of the red. Writing for these intensities $\mathrm{I}_{g}>\mathrm{I}_{r}$, and assuming that the coefficient of transmission of the green is less than that of the red $\mathrm{a}_{\mathrm{g}}<\mathrm{a}_{r}$, it follows that for small thicknesses $I_{g} a_{g}{ }^{\epsilon}$ will be greater than $\mathrm{I}_{r} \mathrm{a}_{r}{ }{ }$, while the reverse will be true for thick layers. This is at once apparent if we call the original intensities of the green and red roo and 50 , and the coefficients of transmission 0.5 and 0.8 , and calculated the intensities of the transmitted colors for several different thicknesses. They will be equal for a thickness $\epsilon$ given by the equation

$$
\mathrm{I}_{g} \mathrm{a}_{g}{ }^{\mathrm{\epsilon}}=\mathrm{I}_{r} \mathrm{a}_{r}{ }^{\mathrm{\epsilon}},
$$

or taking the logarithms of both sides,

$$
\epsilon=\frac{\log \mathrm{I}_{g}-\log \mathrm{I}_{r}}{\log \mathrm{a}_{r}-\log \mathrm{a}_{g}} .
$$

For this thickness the intensities of the red and green. will be equal, and the color of the transmitted light will appear to be yellow, for a mixture of red and green light produces the sensation of yellow when mixed in proper proportions. An excellent mixture for illustrating this can be formed by dissolving "brilliant green" and "naphthalene yellow" in hot Canada balsam and pressing the mixture between two glass plates in the form of an acute prism. The balsam should be previously boiled down until a drop solidifies on cooling, and the dyes should not be added until the fluid has cooled somewhat, otherwise they are apt to decompose. The thin edge of the wedge will appear green, the thick edge red, and 
the intermediate portions, where we have equality of transmission, yellow. If some of the same mixture is moulded into a prism of 20 or 30 degrees angle, the mechanism of dichromatism can be beautifully shown by observing a lamp flame through it. The prism will show the red image well separated from the green, and the latter will be found to be extinguished more rapidly than the former as the prism is moved laterally before the eye.

"Our equations for color show us as well that the color of the transmitted light for a given thickness, will vary with the composition of the original light. If the plate of stained balsam is examined by gas-light and then by day-light, it will be found that parts of it will appear red in the former and green in the latter case. A solution of cyanine and nitroso-dimethyl aniline in alcohol appears red by lamplight and bottle-green by day-light. The same phenomenon is exhibited by the gem Alexandrite, found in the Urals."

Von Bezold" says: "If one takes colored films of different thickness, for instance by piling up several strips of colored glass, the color changes. If the color was originally weak, it becomes stronger and stronger as the number of pieces of glass increases, but the transmitted light becomes weaker and weaker, until finally no light comes through and the layer appears black. If the layer is thick enough, even so-called white or, more properly, colorless glass shows a definite color, usually blue or green. With colored solutions, increasing the concentration has the same effect as increasing the thickness of the layer. ${ }^{2}$ A drop of ink in water colors this bluish or violet according to the nature of the ink, sometimes even greenish or reddish, while the color of the concentrated solution is quite black. With this change in the brightness there is often a change in the color. A solution made from the beautiful blue crystals of copper sulphate is a pale greenish blue when dilute, and deep blue when concentrated. A few

1 "Die Farbenlehre," 54 (1874).

2 [Not strictly true because increasing the concentration may change the dissociation or the polarization. W. D. B.] 
drops of red wine in water colors the water a painful gray and one must add more before one gets the red color of the wine. Similar phenomena are to be noted with plant juices. In thin layers on a white ground madder gives a yellowish Prussian blue to a greenish blue color.

"The red flashed glass colored with cuprous oxide is ordinary glass with a thin superposed film of colored glass; it has a yellowish color when it is put on very thin or when one etches off nearly all of the colored glass. This process is used by people to produce striking chromatic effects on such glasses. The cause of all these variations is that the colored media first absorb certain wave-lengths and then with increasing thickness or concentration gradually absorb others. A series of indigo solutions of varying concentrations has different colors. A very dilute solution appears light blue with a slight touch of green and might easily be mistaken for a solution of Prussian blue. The more concentrated solutions pass through the powerful blue tints to one in which a violet shade cannot be ignored."

It has already been pointed out that we always get both reflection and refraction when a beam of light impinges on a plane, polished surface of an optically denser medium and we have now to consider the factors determining the percentage reflection ignoring for the moment any effect due to actual roughness of the surface. The percentage reflection varies with the angle of incidence, the wave-length, and the polarization of the beam of light, and with the index of refraction and absorptive power of the reflecting medium. Other things being equal, more light is reflected the higher the index of refraction of the reflecting medium and the greater the angle of incidence of the beam. Since the refractive index is usually higher the shorter the wave-length, the percentage reflection will usually increase with decreasing wave-length.

Rays of light exist which possess a one-sidedness and which behave differently when differently orientated." "It is possi-

1 Wood: "Physical Optics," 288, 289, 335, 369 (I9I I). 
ble to obtain light which a glass or water surface refuses to reflect at a certain angle of incidence. Such light is said to be polarized, and is distinguished from ordinary light in that its vibrations are of a fixed type; that is, the ether particle travels in a fixed orbit. If the motion is back and forth along a line, the light is said to be plane-polarized." Light is said to be polarized elliptically when the ether particles move in an elliptical orbit of fixed eccentricity and orientation in space and is said to be polarized circularly when the orbit is a circle. "The discovery was made by Malus in 1810 that light, which had suffered reflection at a certain angle from a surface of water or glass, exhibited the same peculiarities which had previously been observed only in the case of light after its passage through a crystal of Iceland spar .... In general, as we increase the angle of incidence from normal to grazing, the polarization increases, passes through a maximum, and then decreases. The angle at which the polarization is most complete varies with the nature of the substance and is known as the polarization angle. Jamin found that only a few substances with a refractive index of about I.46 polarize the reflected light completely. For all other substances the polarizing angle is merely the angle at which the polarization is a maximum.

"The relation between this angle and the refractive index of the substance was investigated by Brewster who discovered the remarkable law that the index of refraction is the tangent of the angle of maximum polarization. When the light is incident at this angle, the refracted ray makes an angle of $90^{\circ}$ with the reflected ray for

$$
\frac{\sin i}{\sin r}=n=\tan i=\frac{\sin i}{\cos i}
$$

"Therefore $\cos i=\sin r$ and $i+r=90^{\circ}$.

"If this law is true, the angle of maximum polarization will be different for the different colors owing to dispersion. In the case of most transparent media the dispersion is too small greatly to affect the angle as can be shown by examining the image of the sun reflected in a glass plate through a Nicol 
prism so orientated as to cut off most completely the reflected light. The image of the sun appears uncolored, which would not be the case if the angle of polarization was very different for different parts of the spectrum. The Nicol prism takes the place of the second reflector, having the property of cutting off completely light polarized in a certain plane, and transmitting with greater or less facility light polarized in all other planes. In the case of substances having very high dispersion, the variation of the angle with change of wave-length becomes very marked. The organic compound nitroso-dimethyl aniline, which has been found to have the highest dispersion, in the brighter parts of the visible spectrum of any known substance, is adapted admirably for the exhibition of what may be termed the dispersal of the angle of polarization. A little of this substance is fused on a glass or metal plate, or better in a small brass cell heated by steam. A brilliant source of light of small dimensionsnothing is better than a Nernst lamp-is arranged so that its light is reflected from the liquid surface at an angle which can be varied. On examining the light reflected at a fairly large angle with a Nicol prism, it will be found to vary from light blue to deep violet and purple as the angle of incidence is increased, the Nicol being held in such a position as to refuse transmission to the light polarized by reflection."

"When plane-polarized light is twice internally reflected at an angle of $54^{\circ}$, it emerges as circularly polarized light if the original plane of polarization made an angle of $45^{\circ}$ with the plane of incidence. Each reflection in this case introduces a phase-difference of $\pi / 4$ between the reflected components; consequently a single internal reflection from glass will give us elliptically polarized light. This can be shown with an ordinary right-angle prism. In general, when planepolarized light is reflected at an azimuth of $45^{\circ}$, i. e., with its plane of vibration inclined at $45^{\circ}$ to the plane of incidence, the reflected light will be elliptically polarized to a greater or lesser extent. In the case of glass and other transparent media the eccentricity of the ellipse is very great; in other 
words the reflected light is very nearly plane-polarized, but the elliptical polarization is very marked in the case of metals. If plane-polarized light is reflected from a silvered mirror, it will be found to be transmitted quite freely by a Nicol prism in all positions if the plane of polarization originally made an angle of $45^{\circ}$ with the plane of incidence."

"It was found by Jamin and others that in the majority of the cases the light was not polarized completely by reflection at the Brewsterian angle. Moreover, if the incident light was polarized and reflection occurred in the neighborhood of this angle, the reflected light showed traces of elliptical polarization instead of plane-polarized as the formulas indicate. This means that the phase change enters by degrees instead of occurring abruptly at the polarizing angle. In I889 Drude observed that the elliptical polarization produced by a freshly split surface of rock salt was very small, but that it increased rapidly on the exposure of the surface to the air. Shortly afterward Lord Rayleigh found that the ellipticity produced by reflection from water could be eliminated completely by removing the surface film of grease which is always present unless special precautions are taken and which naturally has a higher refractive index than water. These experiments indicate that the disagreement with the formulas is caused by surface films having optical properties different from those of the body of the substance....

"By carefully cleaning the surface of the water Lord Rayleigh was able to destroy all traces of elliptical polarization almost completely, the value of the amplitude ratio being not more than 0.00035 . Quite recently he has found that the positive ellipticity of glass can be changed into negative by repolishing the surface.... Lummer and Sorge found, in repeating and extending the work of Rayleigh, that the elliptical polarization of the light reflected from the surface. of a glass prism was altered by polishing or rubbing one of the other faces or even by subjecting the prism to pressure applied to the bases. The prism was a right-angled one and they found that by rubbing the two base surfaces the ellipticity 
was decreased for all three of the other surfaces, that for hypothenuse passing through zero (the condition for the ideal case of sudden transition) and becoming slightly negative. It is impossible to conceive how the refractive index of a surface film could be altered without touching the surface, much less how it could be changed from a value less than that of the medium to one of greater, as we should be obliged to assume for the conditions found in the case of the hypothenuse surface of Lummer's prism. In view of these experiments it appears more probable that the elliptical polarization is caused in some way by surface strains. At all events further work is required before we can feel justified in establishing a theory. Some unpublished experiments by the author have pointed toward a change in the reflecting power resulting from strains. A very thin film of collodion on glass has been found to more than double its reflecting power under certain conditions. The refractive index of collodion is, however, not very different from that of glass, and we should expect but little reflection from the transition surface. A layer of thick glue spread over a glass surface reduces its reflecting power nearly to zero. When the glue dries, the surface is subjected to a strain so great that glass chips are often torn off the surface. The reflecting power of the surface separating the dry glue and the glass was a little higher than that of a surface between glass and damp glue. The change in the refractive index could hardly account for this as it would be in the wrong direction."

Von Bezold" has called attention to the fact that "the light reflected from a water surface is polarized and if one looks at such a surface through a Nicol's prism or through a suitably arranged Claude Lorraine glass, one can destroy this reflection and can see into the depths. The light of the blue sky is also polarized, the direction and degree of the polarization varying with the relative position of the portion of the sky under consideration to the sun. If the blue sky is reflected in a smooth water surface, the same phenomena

1 "Die Farbenlehre," 84 (I874). 
must take place as with a Claude Lorraine glass. Depending upon the relative positions of the sun, the water, and the observer, the light of the sky may be reflected completely, partially, or not at all from the water mirror. In the last case the actual color of the water is seen and this is why a water surface may appear deep blue or blue-green on days when the sky shows quite pale misty tints. Clouds reflect light irregularly and therefore do not polarize it. The reflection of the light from the clouds is therefore never interfered with at a smooth water surface. For this reason on cloudy days the clearest mountain lakes never show that deep color which charms the observer when the sky is clear.

"The elimination of the polarized light from the sky may give rise to quite startling effects. The author remembers one time in a beautiful summer evening when he walked by the side of a body of water with the sun at his back and the trees along a path were reflected in the water. The trees were seen dark against the light horizon but they appeared light on a dark ground in the reflection. The light scattered in all directions from the leaves showed no definite polarization, and the reflected image was therefore nearly as bright as the original, while the light from the sky was polarized so that it was only reflected slightly from the water surface."

The relation between absorption and reflection is not absolutely clear." "At the beginning of the subject we shall find it convenient to distinguish between two types of absorption: general, in which the absorbing power is very nearly the same for all wave-lengths, at least over a fairly wide range; and selective, when the absorbed region is more or less limited in extent. The absorption of metal films and lampblack represents the first type fairly well. The light transmitted through thin layers differs but slightly in its composition from the original light, and exhibits therefore but little color. Of course there are exceptions, for, as is well known, thin films of gold transmit an excess of green light, while silver is fairly transparent to the ultra-violet. Aniline dyes,

1 Wood: "Physical Optics," 437, 456 (I9I I). 
and, in fact, all colored media, represent the second type, certain colors being freely transmitted, while others are strongly absorbed. When we come to consider the theory of the phenomena, we shall see that the causes of the absorption are radically different in the two cases, though in many cases both conditions may occur simultaneously in one and the same medium."

The light corresponding to certain absorption bands, usually the intense ones, appears for some reason not to enter the substance to any appreciable extent. This light is therefore not absorbed, but is reflected selectively. Stokes ${ }^{1}$ says: "Certain substances, many of them of vegetable origin, have the property of reflecting (not scattering) light which is colored and has a metallic aspect. The substances here referred to are observed to possess an exceedingly intense absorbing action with respect to rays of the refrangibilities of these which constitute the light thus reflected, so that for these rays the opacity of the substances is comparable with that of metals. Contrary, however, to what takes place in the case of metals, this intense absorbing action does not usually extend to all the colors of the spectrum, but is subject to chromatic variations, in some cases very rapid. The aspect of the reflected light, which itself alone would form but an uncertain indication, is not the only nor the principal character which distinguishes these substances. In the case of transparent substances, or those of which the absorbing power is not extremely intense (for example, colored glasses, solutions, etc.), the reflected light vanishes, or almost entirely vanishes, at a certain angle of incidence, when it is analyzed so as to retain only light polarized perpendicularly to the plane of incidence, which is not the case with metals. In the case of the substances at present considered, the reflected light does not vanish, but at a considerable angle of incidence the pencil polarized perpendicularly to the plane of incidence becomes usually of a richer color, in consequence of the removal, in a great measure, of that portion of the reflected light which

1"Mathematical and Physical Papers," 4, 42, 260 (1909). 
is independent of the metallic properties of the medium; it commonly becomes, also, more strictly related to that light which is absorbed with such great intensity. The reflexion from a transparent medium is weakened or destroyed by bringing the medium into optical contact with another having nearly or exactly the same refractive index. Accordingly, in the case of these optically metallic substances, the colors which they reflect by virtue of their metallicity ${ }^{1}$ are brought out by putting the medium in optical contact with glass or water. A remarkable character of metallic reflexion consists in the circumstance, that as the angle of incidence increases from $0^{\circ}$, the phase of vibration of light polarized in the plane of incidence is accelerated relatively to that of light polarized in the perpendicular plane. Accordingly, the same change takes place, with the same sign, in the case of these optically metallic substances; but the amount of the change is subject to most material chromatic variations, being considerable for those colors which are absorbed with great energy, but insensible for those colors for which the medium is comparatively transparent, so that the absorption may be neglected which is produced by a stratum of the medium having a thickness amounting to a small multiple of the length of a wave of light. If the medium be crystallized, it may happen that one only of the oppositely polarized pencils which it transmits suffers, with respect to certain colors, an exceedingly intense absorption; or, if that is the case with both pencils, that the colors so absorbed are different. It may happen, likewise, that the absorption varies with the direction of the ray within the crystal. In such cases the light reflected by virtue of the metallicity of the medium will be subject to corresponding variations, so that the medium is to be regarded as not only doubly refracting and doubly $a b-$ sorbing, but doubly metallic... .

"To illustrate the effect of bringing a transparent medium

${ }^{1}$ I use this word to signify the assemblage of optical properties by which a metal differs from a transparent medium, or one moderately colored, such as a colored glass. 
into optical contact with an optically metallic substance, I may refer to safflower-red. If a portion of this substance be deposited on a glass by means of water, and the water be allowed to evaporate, a film is obtained which reflects on the upper surface a yellowish green light, but on the surface of contact with the glass a very fine green inclining to blue. A green of the latter tint appears to be more truly related to the colors absorbed with greatest energy. Similar remarks apply to the light reflected by Herapathite, according as the crystals are in air or in the mother-liquor. If a small portion of Quadratite (platinocyanide of magnesium) be dissolved on glass in a drop or two of water, and the fluid be allowed to evaporate, the tints reflected by the upper and under surfaces of the film of crystals are related to one another much in the same way as in the case of safflower-red. For a fine specimen of the salt last mentioned I am indebted to the kindness of M. Haidinger. I may mention in passing, that the platinocyanides as a class are of extreme optical interest. The crystals are generally at the same time doubly refracting, doubly absorbing, doubly metallic, and doubly fluorescent. By the last expression I mean that the fluorescence, which the crystals generally exhibit in an eminent degree, is related to directions fixed relatively to the crystal, and to the azimuths of the planes of polarization of the incident and emitted rays...

"I will here mention another instance of the connexion between metallic reflexion and intense absorption. I choose this instance because a different explanation from that which I am about to offer has been given of a certain phenomenon observed in the substance. The instance $I$ allude to is specular iron. As it is already known that various metallic oxides and sulphurets possess the optical properties of metals, there is nothing new in bringing forward this particular mineral as a substance of that kind. It is to the chromatic variation of the metallicity that I wish to direct attention. If light polarized at an azimuth of about $45^{\circ}$ be reflected from a scale of this substance at about the polarizing angle and the reflected light be viewed through a plate of calcareous spar and 
a Nicol prism, it will be found, by using different absorbing media in succession, that the change of phase, as indicated by the character of the rings, while it is very evident for red light, becomes much more considerable in the highly refrangible colors. Now specular iron is almost opaque for light of all colors, but as it gives a red streak it appears that the substancecolor is red; and, in fact, it is known that very thin laminae are blood-red by transmitted light. Accordingly, the chromatic variation of the change of phase corresponds to that of the intense absorbing power.

"The light reflected by specular iron is not extinguished by analyzation, whatever be the angle of incidence; but at the angle of incidence which gives the nearest approach to complete polarization, a quantity of blue light is observed to remain. This has been explained by comparing specular iron to a substance of high dispersive power, so that the polarizing angle for red light is considerably less than for blue; and accordingly on increasing the angle of incidence, the light (which is here supposed to be analyzed so as to retain only the portion polarized perpendicularly to the plane of incidence), while it becomes much less copious near the polarizing angle, becomes at the same time of a decided blue color. ${ }^{1}$ I believe, however, that the blue light is mainly due to the chromatic variation of the metallicity, the medium, considered optically, being much more metallic for blue light than for red, though it may in some measure be due to the cause previously assigned.

"Specular iron is a good example of a substance forming a connecting link between the true metals and substances like safflower-red. It resembles metals in the circumstances that the absorbing power, as inferred from the chromatic variation of the metallicity, and as indicated by the tint of the streak, is not subject to the same extensive chromatic variations as in the case of coloring matters like safflowerred. It resembles safflower-red in being sufficiently transparent with respect to a portion of the spectrum to allow the

${ }^{1}$ See Dr. Lloyd's "Lecture on the Wave-Theory of Light," Part II, p. 8. 
connexion between the metallicity and the substance-color to be observed; whereas the substance-color of metals is not known from direct observation, except, perhaps, in the case of gold, which in the state of gold-leaf lets through a greenish light."

"The instances in which substances appear colored by reflexion are comparatively rare. It is very common in chemical descriptions to read of a solution appearing of such a color by transmitted, and such a color by reflected light. In many cases, that is a positive mistake, and the color described as due to reflexion is really due to transmission. A chemist views a solution contained in a test-tube by transmission, and then by reflexion; and seeing, perhaps, some perfectly different color in the latter case, describes it as the color of the solution by reflexion, whereas it is merely the color by transmission due to a greater thickness, the light having been reflected at the back or bottom of the test-tube, and so having twice passed through the solution. In other cases the color described as due to reflexion really arises from fluorescence; and though the statement may be true in the sense intended, it seems objectionable to apply the term reflexion to a process so utterly different. It is only in the case of metals, such as gold and copper, and of certain other substances such as murexide, platinocyanide of magnesium, etc., that color is really seen as the result of reflexion....

"When this takes place in the case of non-metallic substances, they are found to be endowed, for the colors so reflected, with an intense opacity, comparable with that of metals; while for other parts of the spectrum they may be comparatively transparent, and these parts they reflect with an energy comparable to that of a vitreous substance only. The variations of absorbing power in passing from one part of the spectrum to another, and consequently the variations in reflecting energy, are frequently much more considerable, and accordingly the color by reflexion is much richer than in the case of metals.

"An excellent example of the intimate connexion between 
metallic reflexion and intense absorption is afforded by crystals of permanganate of potash. These crystals exhibit a green metallic reflexion, and when, crushed yield a powder of an intense purple color by transmitted light. The color is too intense for spectral analysis but the solution has a similar color, merely less intense as corresponds with its smaller concentration, and the analysis of the light transmitted by the solution presents no difficulty. The green is quickly absorbed, but when the solution is sufficiently dilute, five eminently characteristic bands of absorption are seen in that part of the spectrum. A sixth band comes out with a greater thickness or else less strength of solution, but even the fifth is somewhat less strong than the others. When the light reflected from a crystal is analyzed, four bright bands are seen standing out on a generally luminous ground of inferior brightness. These bright bands correspond in position with the principal dark bands in the light transmitted by the solution, and therefore, it may be presumed, by the crystals themselves. When the angle of incidence has a suitable value, and the reflected light is analyzed by a Nicol's prism, with its principal plane in the plane of incidence, and then by a common prism, the spectrum is reduced to these four bright bands. A fifth bright band could perhaps be made out, in the case of a fine crystal with a fresh surface. Under the circumstances described, the Nicol's prism would extinguish the light reflected from a vitreous substance, and transmit much of that reflected from a metal. We see, therefore, that, as regards its relations to light, the crystallized body passes repeatedly from the condition of a vitreous to that of a metallic substance and back again, as the refrangibility of the rays, in relation to which it is considered, is continuously increased by a small amount.

"The same relation between intense absorption and metallic reflexion exists generally, though it cannot be always studied by means of a solution. The platinocyanides, for example, yield colorless solutions, so that the intense absorption which most of them exercise for certain parts of the 
spectrum must be attributed to the mode in which the molecules are built up in forming the crystals; but by attending to the color of the light transmitted by thin crystals, the law is found to be obeyed. Gold can only be obtained, in solution, as gold, by means of the opaque solvent mercury; but its color by transmission may be studied in gold leaf, or in a chemically deposited film, and is then found to be conformable to the law mentioned, the less refrangible colors, which are those which are the more copiously reflected, being also those which are the more intensely absorbed.

"When a body endowed with the property of colored reflexion, such as permanganate of potash, is dissolved, in consequence of the necessary dilution the opacity of the medium ceases to be, for any part of the spectrum, of that intense kind which is necessary for quasi-metallic reflexion; and accordingly the light reflected by the solution is colorless. Hence colored reflexion is not available for following a substance through mixtures containing it. The chemist ought, however, to be acquainted with its laws, in order to understand the changes of color which a substance possessing the property is capable of exhibiting in the solid condition, according to its state of aggregation.

"In order that the color due to reflexion should appear, it is necessary that the substance should have a certain amount of coherence. Thus indigo in the form of a fine loose powder is blue, even when viewed by reflexion. It would be erroneous, however, to describe the body as blue by reflexion, if we were speaking of the properties of the substance, and not the mere crude results of observation made under given circumstances. For though it is true that the light by which the blue color is seen has undergone reflexion (without which it would not have reached the eye) it is not in reflexion that the chromatic selection is made by virtue of which the powder appears blue, but during transmission. In fact it is only a small portion of the light that is reflected at the outer irregular surface of the mass; the greater part penetrates a little way, and is reflected at various depths, and in passing through 
the particles, in going and returning, suffers absorption on the part of the colored substance. Were the substance intensely opaque for all the colors of the spectrum, the powder would be not blue but black, as we see in the case of platinum-black. By burnishing, the powder is reduced to the state of a somewhat coherent mass, and it now begins to exhibit the copper color due to reflexion. The internal reflexions are at the same time greatly weakened, so that the part of the light which is reflected from beneath and undergoes absorption is much reduced. A pressed mass is not, however, an optically homogeneous medium, so that the color by reflexion obtained by burnishing cannot in general be quite pure. In the state of a fine crystalline powder, indigo exhibits a mixture of the copper color due to reflexion, and the blue color due to transmission, though observed in the light reflected from the mass as a whole; while if the substance could be obtained in large crystals, the color by reflection would be seen in perfection, and the color by transmission would disappear, the crystals being sensibly opaque."

Wood $^{1}$ says that substances which reflect selectively exhibit surface color. "The aniline dyes are excellent examples. A dye which in solution absorbs green light; appearing purple by transmitted light, in the solid state reflects green light selectively. Absorption, however, is not the only factor which determines this selective reflection, and we often find misleading statements in text-books on optics, it being frequently stated that the wave-lengths most copiously reflected are the ones most strongly absorbed. This is by no means the case. Cyanine, for example, has a strong absorption band in the yellow, while the color of the selectively reflected light is purple, not so very different in hue from that of the transmitted light. If we examine the spectrum of the reflected light, we find a very dark band in the green, the centre being not far from wave-length 0.0005 . The distribution of intensity in the rest of the spectrum is not very different from what it would be in the case of reflection from

1 "Physical Optics," 440, 409, 417, 400 (IgII). 
glass, which shows that the peculiar color of the dye is not so much due to a very powerful reflection of certain waves as it is to its almost complete, refusal to reflect a certain region of the spectrum."

"Not all absorption bands exhibit the phenomenon of quasi-metallic reflection, and it is of interest to inquire into the conditions governing its appearance. Absorption results from resonance vibration of the electrons, but in some cases they throw practically all of the energy back, and there is little or no true absorption. When we come to the consideration of metals, we shall see that there is a type of absorption resulting from the presence of free electrons which have no definite period of vibration. If these electrons are free to move without friction under the periodic forces of the light-waves, the electric intensity within the medium will be neutralized within the medium and we shall have complete reflection. This would be the case for a perfect conductor. If the electrons experience a resistance to their motion, the neutralization will not be complete and a portion of the energy will enter the medium and be absorbed or transformed into heat (ohmic heating). It is the surface layer only which contributes to the reflecting power; in other words, the resonators must be so densely packed that the wave is practically stopped within less than a wave-length of the surface. That this is so follows from the principle of interference. Suppose each layer of resonators to reflect a small percentage of the incident energy. The phases of the disturbances would not be in agreement; and they would destroy each other. Now we find that ultra-violet absorption bands, say those of glass or quartz, do not give rise to selective reflection. From this we should infer that the disturbance penetrates a short distance into the medium and is absorbed. Thin films of glass, only a few wave-lengths in thickness, are quite transparent, even to the extreme ultra-violet. They would, without doubt, be found to be absolutely opaque to the infra-red at $8.5 \mu$. It is possible to obtain scales of mica less than a wave-length thick, and it would be interesting to 
compare their transmission at $8.5 \mu$ and in the remote ultraviolet, say at $0.2 \mu$. The absorption bands of many substances, both in the infra-red and visible region, are of the same nature as these ultra-violet bands, and cannot therefore be located by the method of residual rays. Selenite and alum belong to this class, as was found by Aschkinass. In some cases traces of selective reflection can be found by employing only a single surface. The residual rays are in this case much diluted with continuous spectrum, but they can be located if due precautions are taken. This method was adopted by Coblentz, who investigated the reflecting power of a large number of substances. Obviously in cases where the reflecting power is below 70 per cent, most of the energy would be lost after four or five reflections. For 50 per cent reflecting power, four surfaces would only give us $1 / 16$ of the total intensity of a band of residual rays....

"The selective reflection of powdered quartz in the infra-red has been investigated by. A. Trowbridge. $\mathrm{He}$ found a minimum of reflecting power in a region of the spectrum corresponding to the absorption band at $2.95 \mu$, and a maximum at the absorption band at $8.5 \mu$. This is interesting, and shows us the difference in the behavior of the two types of absorption bands. The one at $2.95 \mu$ is weak, and only manifests itself when a considerable thickness is traversed. The quartz therefore shows 'body color' by reflection, i. e., a color resulting from absorption, the energy penetrating deeply into the powdered mass, and finally emerging as the result of repeated reflection and refractions among the particles, robbed of energy of wave-length $2.95 \mu$. The band at $8.5 \mu$ is metallic in character, and the energy is selectively reflected from the upper surfaces of the particles. If the particles were very small and the surface flat, specular or regular reflection would appear. Trowbridge used a rather coarse powder, and studied the diffuse reflection. The distinction between the two types of bands must be taken into account in all investigations made with surfaces which permit of the formation of 'body color,' otherwise the results will appear to be very anomalous in character." 
"Very little" work has been done on selective reflection in the ultra-violet. In general the phenomenon of metallic reflection does not occur at the ultra-violet absorption bands. Some investigations, made by Martens, of the ultra-violet dispersion of certain organic compounds, indicate that tiaces of selective reflection exist in certain cases. Cassia oil, for example, which in the thinnest possible films, pressed between fluorite plates, is opaque to everything below wave-length 3360 , exhibits the phenomenon. Light from the cadmium spark, reflected a number of times from its surface, is shown by the spectroscope to consist chiefly of the 2748 lines, no trace of the equally strong lines 2573 and 2321 appearing on the photograph. Fourfold reflection from a surface of monobromnaphthalene showed the lines $232 \mathrm{I}$ and 2288 only. Bisulphide of carbon, investigated by Flatow, showed a reflection maximum at 23 Io. This is a subject which requires further investigation, and the work already done should be repeated. In working with ultra-violet rays, we are completely in the dark, and the high dispersion of all transparent substances in this region means wide angular separation of the rays at any refracting surfaces used. Great care must be taken to make sure that the rays of all wave-lengths have a chance to reach the spectroscope. Quantitative measurements of the reflecting power for different wave-lengths should be made at the same time and the transmission of the thinnest possible films examined. If the substance is in contact with glass, quartz, or some other transparent substance, we may have a selective reflection resulting from the difference between the values of the refractive index. This will modify the color of the reflected light in any case. In many cases the color of the light reflected is totally different from that most strongly absorbed. For example, the surface color of cyanine is purple, and the absorption band is in the yellow. Its purple color is due to its failure to reflect a region of the spectrum bordering the absorption band on the green side, resulting from the very low value of the refractive index. In contact with glass, viewed from the glass side, it 
reflects yellowish green light. Nitroso-dimethyl aniline, which has an absorption band in the violet, exhibits a brilliant blue reflection when in contact with glass (from the glass side), but practically no trace of color from the surface in contact with air. Selective reflection of this nature is chiefly the result of the high value of the refractive index, and cannot be used as a method of locating the position of the absorption band. Such experiments show us the care we should use in interpreting results found in the ultra-violet, where effects due to refractive index may be even greater."

"Stibnite, or natural sulphide of antimony, is a substance which exhibits very remarkable optical properties. It has a metallic lustre resembling that of freshly cut lead, and is opaque to visible light. Nevertheless, as Coblentz has shown, it is extremely transparent to the infra-red radiations, a slab $0.4 \mathrm{~mm}$ in thickness transmitting 46 per cent of the energy out to $12 \mu$ while one $4.9 \mathrm{~mm}$ thick allowed 43 per cent to pass. This shows us that the absorption is very nearly nil, and that the rest of the energy is reflected at the surface. The reflecting power of the substance is about 37 per cent, and results from the very high value of the refractive index, which is 5.53 for blue light and 4.69 for red, according to the calculations of $\mathrm{E}$. C. Müller, made from observations of the constants of elliptical polarization. Measurements made by the author with a Michelson interferometer, and by observing Newton's rings, with a film of stibnite obtained by cathode deposition, give the value 3 for yellow light, the lower value doubtless resulting from a lightly spongy condition of the deposit, which exhibited a lower reflecting power than the cleavage surfaces of the mineral."

Rayleigh ${ }^{1}$ has discussed the particular question of reflection by metals. "The peculiarity in the behavior of metals towards light is supposed by Cauchy to lie in their opacity, which has the effect of stopping a train of waves before they can proceed for more than a few wave-lengths within the medium. There can be little doubt that in this

${ }^{1}$ Phil. Mag., [4] 43,32 I (1872). 
Cauchy was perfectly right; for it has been found that bodies which, like many of the dyes, exercise a very intense selective absorption on light, reflect from their surfaces in excessive proportion just those rays to which they are most opaque. Permanganate of potash is a beautiful example of this, given by Professor Stokes. He found that when the light reflected from a crystal at the polarizing angle is examined through a Nicol held so as to extinguish the rays polarized in the plane of incidence, the residual light is green, and that, when analyzed by the prism, it shows bright bands just where the absorption spectrum shows dark ones. This very instructive experiment can be repeated with ease by using sunlight, and instead of a crystal a piece of ground glass sprinkled with a little of the powdered salt, which is then well rubbed in and burnished with a glass stopper or otherwise. It can without difficulty be so arranged that the two spectra are seen from the same slit one over the other, and compared with accuracy....

"That a sufficient opacity is as competent as a high optical density to produce an abundant reflection is evident without any analysis. So long as the medium into which the light seeks to penetrate remains nearly at rest, the greater part of the motion must be thrown back without any regard to the cause of the approximate acquiescence. Whether the sluggishness be due to a great inertia or a correspondingly great friction is in this respect of no importance. In order, however, to account for the reflection from silver ( 90 or 95 per cent) without opacity, a very high optical density would be required, much higher than we have any reason to think at all likely. On the other hand, we know that the opacity of metals to light is very great.

"In this connection it is interesting to note that some, and probably many, non-metallic substances possess a quasimetallic reflecting power for dark radiation. De la Provostaye and Desains long ago remarked on the large percentage of dark heat reflected from glass, which was much in excess of that calculated from Fresnel's formulas with the known refractive index. The observation seems to have remained 
uninterpreted; but we cannot well be wrong in attributing the extra reflection to an opacity to the rays of dark heat, which, always great, rises somewhere in the spectrum to such a magnitude as to damp the entering rays within a few wavelengths of the surface. Nothing but direct experiment can inform us what substances are sufficiently opaque to exercise an abnormal reflection; for the stoppage of radiant heat by a plate of ordinary thickness may well be complete to sense, and yet not sufficiently sudden to give any material assistance in reflection. I am glad therefore to be able to refer to the experiments of the late Professor Magnus, in which he investigates the proportion of heat reflected by plates of various substances, the incident radiation being derived from moderately heated plates of the same or of a different material.

"First let us see what fraction of the incident radiation (unpolarized) would be reflected from the surface of a substance having a refractive index of 1.5 -about that of glass. If $Q$ be the angle of incidence, and I the corresponding fraction, I find by calculating from Fresnel's formula the following:

$$
\begin{array}{llll}
Q=0^{\circ} & Q=33^{\circ} & Q=45^{\circ} & Q=62^{\circ} \\
I=0.040 & I=0.042 & I=0.050 & I=0.100
\end{array}
$$

This is for one surface. If the plate be quite transparent the reflection may be nearly the double of the above. Now for glass at an angle of $45^{\circ}$. Magnus found no smaller value of I than 0.084 ; and as this must be attributed almost, if not entirely, to the first surface, it is clear that something not taken account of in Fresnel's theory must have come into operation. But by far the most remarkable result was with fluor-spar for the reflecting, and rock-salt for the radiating plate. The reflection at $33^{\circ}$ was no less than 0.23 , at $45^{\circ}$ it was 0.242 and at $62^{\circ}$ it was 0.335 ; and to this the second surface cannot contribute sensibly. Unquestionably, therefore, the reflecting power of fluor-spar for a certain kind of dark radiation is greatly in excess of what can be accounted for without an extreme opacity, a result which is the more remarkable because for dark radiation in general fluor-spar is one of the most transparent things known. The reflection from 
a plate of rock-salt was found to be much the same as from glass; but here, I presume, we may consider both surfaces to be operative, in which case the result is normal. ${ }^{1}$ It is curious that opacity first diminishes the reflection from a plate, and then, when extreme, increases it again and that without limit."

Wood ${ }^{2}$ also discusses the question of metallic reflection. "While metals possess in general a high reflecting power, a considerable portion of the incident energy penetrates the surface and is absorbed. In the case of steel, for example, nearly one-half of the light is lost by absorption, while even silver absorbs 5 per cent. In the majority of cases the absorption is general, that is, it is not confined to a narrow range of wave-lengths, as is the case with the substances which we have examined thus far. It is to a certain extent selective, however, as is best illustrated by gold-leaf, which appears green by transmitted light, and thin films of chemically deposited silver, which appear blue. These silver films are fairly transparent to a limited range of ultra-violet radiations, which lie just beyond the limit of the visible spectrum. It is probable that the absorption of metals is due to the presence of two types of electrons-conducting ones which are free to move indefinitely under the influence of a steady electric force, and non-conducting ones which are similar to those which we have considered."

"The laws which govern the optical behavior of metals are very different from those which hold in the case of transparent substances and substances showing strong selective absorption. Metals exhibit in general a very high reflecting power, and in some cases, also a powerful absorbing action. We must distinguish carefully between absorption and reflection. If we examine a thin film of silver deposited on glass, we find that it transmits little or no light, and we might there-

${ }^{1}$ According to the experiments of Masson and Jamin, the transmission of a perfectly transparent plate is always about 92 per cent, whether the material be glass, rock-salt, or alum. This is in agreement with the calculation in the text, as about 8 per cent would be reflected.

2 "Physical Optics," 449, 456 (I9II). 
fore come to the conclusion that the metal absorbs strongly. A little further investigation will show, however, that over 90 per cent of the light has been reflected, the remaining Io per cent having been absorbed. Platinum, however, has a much lower reflecting power, combined with equally great opacity; consequently we may regard platinum as possessing a stronger absorbing power than silver. Gold is, however, as compared to silver and platinum, extremely transparent, ordinary gold-leaf transmitting no inconsiderable amount of green light.

"While metallic absorption is not in general characterized by such marked selective action as is the case with the colored media which we have studied, the phenomenon of selective absorption is by no means absent, as the strong coloration of the light transmitted by gold-leaf proves: silver, too, while it appears to favor equally all wave-lengths in the visible spectrum, is fairly transparent to ultra-violet radiation comprised within the range $0.305-0.320$ : its reflecting power is correspondingly low for these same wave-lengths.

"The colors which metals exhibit are due to a selective reflecting power, which is especially marked in the case of gold and copper. If two gilded glass plates are mounted in parallel at a distance of a centimeter or so, with the reflecting surfaces opposed, and a beam of light caused to travel back and forth between them, suffering a number of reflections, the surface color is still more marked, the filament of an incandescent appearing as red as if seen through ruby glass. This method of bringing out the surface color is analogous to the method of Rubens and Nichols for isolating long heat waves by multiple reflections from surfaces of quartz and rock-salt. The same phenomenon can be seen in less marked degree by looking into the interior of a gilded goblet, the bottom of which appears of a fairly deep-red color. Glass plates can be easily gilded by exposing them to the discharge from a gold cathode in a vacuum tube; plates gilded by the application of gold-leaf, in the manner employed for lettering on 
windows, would doubtless answer as well, and can be readily obtained from a sign-painter.

"The phenomenon of elliptical polarization is exhibited in a high degree when light polarized in an azimuth of $45^{\circ}$ is reflected obliquely from a metal surface. In the case of transparent substances, we have seen that this result can occur if the surface is contaminated with a film, but in the case of metallic reflection the presence of a surface film is not necessary."

The ratio of the intensity of the reflected light to that of the incident light at normal incidence is called the coefficient of reflection, R. For metals we have the equation

$$
\mathrm{R}=\frac{n^{2}\left(\mathrm{I}+k^{2}\right)+\mathrm{I}-2 n}{n^{2}\left(\mathrm{I}+k^{2}\right)+\mathrm{I}+2 n}
$$

where $k$ is the extinction coefficient. ${ }^{1}$ In the case of metals $2 n$ is small in comparison to $n^{2}\left(\mathrm{I}+k^{2}\right)$. If we neglect it, we get $R=I$. In the case of silver 95 per cent of the incident light is reflected, $i$. e., $\mathrm{R}=0.95$. The reflecting power will be greatest for the wave-lengths for which $k$ has its greatest value; thus gold reflects red light much more powerfully than green. The best determinations of the reflecting power of different metals are those made by Rubens and Hagen. ${ }^{2}$

It is apparent from their results that the reflecting power of silver in contact with glass is somewhat less than that of silver in contact with air. The same is true for mercury.

"An easy way of exhibiting the loss of light by reflection from a metal is to half fill a test-tube with mercury and plunge it in a jar of clean water; the light reflected from the metal will appear quite dull in comparison with the light totally reflected at the glass-air surface.

"The values of $\mathrm{R}$ calculated from observations by katoptric methods (yellow light) were found in rough agreement with the values observed by Rubens. In the case of silver

1 Wood: "Physical Optics," 465, 440, 439 (I9II).

2 Drude's Ann., I, 352 (r900); 8, I (I902). 
the minimum at $\lambda=3 \mathbf{I} 6$ is very remarkable, the reflecting power of the metal for this wave-length being about that of glass for yellow light. The highest reflecting power appears to be possessed by metallic sodium, for which $\mathrm{R}=99.7$ according to Drude. This value was calculated, however.

"The most recent investigations of the reflecting and absorbing power of metals has been made by Hagen and Rubens. The films were deposited upon quartz plates, which permitted of investigations in the ultra-violet region, and their thickness determined by two different methods, weighing the film both before and after its conversion into the iodide and the interference method of Wernicke. They first investigated the dependence of reflecting power upon the thickness. From the data for gold we see that the reflecting power increases with the thickness, reaching its maximum value at about $80 \mu \mu$ or $0.00008 \mathrm{~mm}$, which is about one-eighth of the wave-length of red light, after which it remains constant. For red light this maximum value is 90 per cent; for green light it is less than 50 per cent. This explains the yellow color of gold and the green color of gold-leaf by transmitted light. In the ultra-violet it is necessary to use somewhat thicker films to get the full value of the reflecting power."

"In the case of absorbing media, the reflecting power depends both upon the refractive index and the coefficient of absorption. Now, absorbing media have a high refractive index on the red side of the absorption band and a low index on the blue side, consequently the spectrum of the reflected light will be brightest on the red side of the absorption band, since for these wave-lengths we have a large coefficient of absorption and a high refractive index. On the blue side, however, the low value of the index diminishes the reflecting power more than the augmentation due to the powerful absorption. The hue of the surface color thus depends on the refractive index of the medium in which the substance is immersed, for it is the relative and not the absolute refractive index in which we are concerned. Cyanine in contact with glass exhibits a yellowish green surface color, much more nearly 
resembling the hue of the absorbed light. If the dye could be brought in contact with a transparent substance having the same dispersion, the wave-lengths selectively reflected would be identical with those absorbed, since in this case the relative refractive index would be unity for all wave-lengths.

"An excellent way of showing the variable reflecting power of a film of cyanine is to compare it with glass in different parts of the spectrum. A little of the melted dye is pressed between two plates of hot glass, which are separated when cold. A spot is selected where the film has a good optical surface, and this spot is left on the glass, the rest being cleaned off. By holding the plate in the spectrum formed by a prism or grating, the reflecting power of the two surfaces can be studied. In some parts of the spectrum the cyanine reflects more strongly than glass; in other regions the reverse is true, while at wave-length 0.0005 the cyanine refused to reflect to such a degree that the film appears as a black spot on the blue field reflected by the glass.

"It is interesting to note the difference in the surface color of the dye when the reflection takes place at the surface in contact with the glass. A very convenient way of showing the yellowish green color in this case is to press out a film of the molten dye on one surface of a prism of 8 or Io degrees angle. ${ }^{1}$ In this way the light reflected from the dye can be obtained uncontaminated with the light reflected from the glass surface. The method is analogous to that employed by Lippmann in mounting his color photographs. The calculation of the curve of reflected intensities under these conditions makes a good exercise for the student."

"The colors of most natural objects result from absorption. The light penetrates their surfaces and then suffers internal

${ }^{1}$ A suitable prism can be made in half an hour by grinding down a piece of thick plate window glass. A strip of thick glass cemented along one edge will be all that is necessary to make the glass take the required form. Grind on a piece of glass with a very coarse emery at first, then use finer grades, polishing with rouge at the end. Small scratches do no harm, and a high polish is not necessary. 
reflections or refractions and emerges robbed of the rays which are most strongly absorbed. If this is to happen, it is clear that the substance must not be homogeneous, otherwise the reflections and refractions, which return the unabsorbed light, will not occur. It is thus incorrect to say that colored pigments reflect certain colors more strongly than others: If the pigment particles formed a continuous and homogeneous medium, no color whatever would appear in the reflected light, which would be white. If any color appeared, as it might if the pigments were a very powerful absorbent, it would be the tint complementary to the one exhibited by the powder.

"Since pigments produce color by absorption, it is at once apparent why a mixture of two pigments does not exhibit the color which we should obtain if we actually mixed the colored lights which they appear to reflect. The light reflected from the mixture is the residual color which remains after the dual absorption has taken place. For example, if we mix yellow light and blue light, we get white, while a mixture of a blue and yellow pigment appears green. The reason of this is, that the yellow pigment absorbs the blue and violet, the blue pigment the red and yellow, the mixture absorbing everything except the green."

Von Bezold ${ }^{1}$ has called attention to the difference between colored substances which show selective absorption only and those which show selective reflection also. "Only in certain types of substances is the color due to absorption. There are other substances in which certain rays penetrate and certain others are reflected. Such substances have different colors in reflected and in transmitted light. The light which is reflected at the surface is colored and these substances are therefore said to have surface colors. The metals are typical of these substances. Very thin goldleaf is a beautiful blue-green by transmitted light, and very thin films of silver, such as one gets when making mirrors,

1 "Die Farbenlehre," 72 (I874). 
are deep blue, while the mirror image of a white substance as seen on a golden surface is yellow, and orange-yellow after many reflections, while the image from a silver mirror is yellowish white. Many aniline colors show similar surface colors in the dry state. If one lets a small amount of a solution of one of these colors dry on a glass plate, the film appears completely metallic. For instance, magneta is a greenish gold color by reflected light and purple-red by transmitted light. Of the pigments used in painting very few show these colors and only to a slight extent. In a solid stick, indigo shows a slight trace of copper red and so does Prussian blue, though to a lesser extent."

Cornell University 